\title{
Design of a Multi-body Pipe Inspection Robot
}

\author{
$1^{\text {st }}$ Cuautli Garcia \\ School of Electrical Engineering \\ and Computer Science \\ University of Ottawa \\ Ottawa, Canada \\ cgarc074@uottawa.ca
}

\author{
$2^{\text {nd }}$ Eric Lanteigne \\ Department of Mechanical Engineering \\ University of Ottawa \\ Ottawa, Canada \\ eric.lanteigne@uottawa.ca
}

\author{
$3^{\text {rd }}$ Wail Gueaieb \\ School of Electrical Engineering \\ and Computer Science \\ University of Ottawa \\ Ottawa, Canada \\ wgueaieb@uottawa.ca
}

\begin{abstract}
This paper presents a new hypermobile robot for inspecting pipes of different diameters. The robot is composed of three modules, two driving modules and one control module which are linked by a passive joint. The driving module has eight actuators: four gearmotors to propel the robot along the pipe, and four servomotors to control the radial position of the robot in the pipe and to maintain the robot's balance. A Raspberry $\mathbf{P i}$ is used to control the actuators, acquire sensors feedback, and receive commands from a remote wireless user-controlled GUI. A set of preliminary experimental tests was conducted to demonstrate the effectiveness of the system.
\end{abstract}

Index Terms-Inspection robot, in-situ pipe inspection, hypermobile robot

\section{INTRODUCTION}

As a result of string growth in U.S. and Canadian oil and natural gas production, pipeline capacity is expected to become constrained in the future, requiring new pipelines and pipeline expansions to provide access to new markets. There are more than $840.000 \mathrm{~km}$ of transmission, gathering and distribution pipelines in Canada, according to the Natural Resources Canada. These pipelines are aging and increasing demands posed by harsher service conditions stress the importance of integrity management [1]. As such, leakage detection and maintenance are crucial.

Generally, a pipeline is a network of connected pipes with pumps, valves and control devices to help convey liquids or gases. A pipeline network consists of gathering systems, trunk links and distribution systems. The latter is the longest of the network. Typically, the pipes making the distribution system are of a small diameter and operate at low pressure.

Pipelines operate year round and computerized operation allows pressure, flow and energy consumption throughout the line to be continuously monitored. Software can perform leak detection calculations quickly and initiate remedial actions in case of emergency. However, research suggests that operating stations identify only about $15-20$ percent of the total pipeline leaks [2].

According to the Canadian Energy Pipeline Association, sophisticated technology allows operators to see anything out of the ordinary, like minute cracks or signs of corrosion, from inside the pipe. Like a small submarine, devices called smart pigs (pipe inspection gauge) are used for inline inspection. These devices are not autonomous. Rather, they move along with the fluid. An autonomous robot is a widely accepted method for collecting data, including images, of pipes without or with little human intervention. To this end, several autonomous mobile robots for small diameter pipe inspection have been proposed [3], [4].

The goal of this research is to validate the theoretical robot design presented in [3], [4]. The proposed mobile robot design is comprised of three modules: two propulsive modules and a control module. It can travel inside pipelines between 154 and $203 \mathrm{~mm}(6 "$ and 8") in diameter and negotiate bends of 0 to $180^{\circ}$.

This article describes the mechanical systems developed to achieve the desire motion capabilities and the implementation of the electronic controller architecture. The remainder of the paper is organized as follows: Section II explores existing inpipe robot morphologies. Section III describes the theoretical and computer-aided design of the proposed robot. Section IV outlines the electronic system implementation. Experimental results are provided and discussed in Section V, followed by a few concluding remarks and suggestions for future research.

\section{RELATED WORKS}

Most in-situ pipe robots have been designed for specific applications and can be classified into several elementary categories according to their locomotion, as shown in Fig. 1.

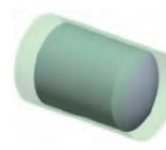

(a) Pipeline Pig

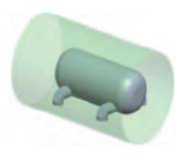

(e) Crawl Type

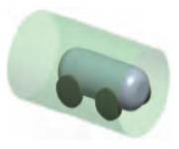

(b) Wheel Type

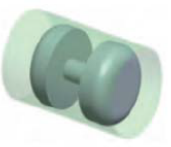

(f) Creep Type

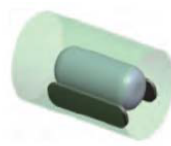

(c) Crawler Type

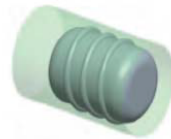

(g) Screw Type

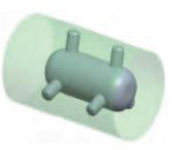

(d) Support Type

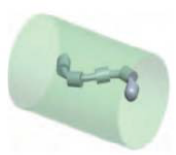

(h) Snake Type
Fig. 1. Classification of in-pipe robots [5] 
The pig type illustrated in Fig. 1(a) is one of the most wellknown commercial in-situ pipe robots. It is passively driven by the fluid pressure and, as a consequence, cannot execute sophisticated movements. Okamoto et al. developed a pig type robot that has a cylindrical capsule which is connected to rubber discs by passive joints which allows the capsule to rotate around its longitudinal axis. The principal purpose of the rubber discs is to block the fluid and to propel the robot; however, the discs maintain the radial position of the capsule. It is also equipped with multiple ultrasonic sensors directed to the pipeline to analyze the inner wall of the pipeline [6].

The wheeled in-situ pipe robots, shown in Fig. 1(b), can move smoothly and fairly quickly along horizontal pipes due to the convenience of wheel-based locomotion. However, they cannot operate inside vertical or inclined pipes. Round ducts or pipes which can typically be represented as a cylindrical workspace are the most common geometrical shapes, and thus wheeled robots working in round ducts/pipes are the most common practices [7]. As an example, Song et al. developed a controller for guiding wheeled mobile robots inside cylindrical workspaces. The prototype is a car-like mobile robot with wheels of $120 \mathrm{~mm}$ of radius. The robot was programmed to navigate with a low speed of $3 \mathrm{~m} / \mathrm{min}$ inside $250 \mathrm{~mm}$ radius pipes [8].

Crawler or Caterpillar type robots, illustrated in Fig. 1(c), are similar to wheeled robots but are characterized with a higher traction which can be useful in certain conditions, such as slippery surfaces or inclined pipes. Nonetheless, they still cannot propel themselves in vertical pipes [9].

Support or Wall-press robots are depicted in Fig. 1(d). They can adapt their structure to pipes of varying diameters, but they usually need another propulsive module to provide the steering movement inside fittings. The structure of this type of robot is suitable for long-range inspection and it can also carry heavy loads [10]. The robot presented by Kwon et al. can inspect a pipeline between 80 and $100 \mathrm{~mm}$. It uses two driving modules connected by a compression spring. The modules are offset by $60^{\circ}$ to help with the robot's agility. Each module is constructed as a triangular linkage structure to maintain its alignment with the pipe while having a caterpillar wheel on each side of the triangular structure. The caterpillar wheel is made of two gears and a wrapping silicon belt for a large friction coefficient [11].

Kin et al. developed a robot that is somewhere between crawler and support type. The robot can locomote in pipes between 600 and $800 \mathrm{~mm}$. It uses pneumatic cylinders to adjust to the pipe surface while having tracks along the robot [12].

A crawl type robot, illustrated in Fig. 1(e), is able to move through horizontal and bending pipes. However, it cannot locomote along vertical or inclined pipes. Yu et al. developed a walking robot for horizontal pipes that uses a support platform with four passive wheels connected to the robot by springs to adapt itself to different diameter pipes. The propulsion of the robot is realized by 14 gears transmitting the motor's motion into two axes that rotate at different rates [13].

A creep type robot is shown in Fig. 1(f). It is reliable for inspecting vertical/inclined pipes because it is capable of applying a great force to the pipe wall. It can also adapt to different diameter pipes. This robot usually has two modules connected by a joint that can rotate and separate both modules. Zhang et al. presented this type of structure as a squirm pipe robot with magnetic wheels that use the creep principle to navigate along the pipe [14]. Another creep robot presented by Kejie et al. is able to move in horizontal, vertical and bending pipes while adapting 90 to $150 \mathrm{~mm}$ diameter pipes. Nevertheless, it is not autonomous. As a matter of fact, it must be tethered to the controller and the batteries [15].

The screw drive in-pipe robot (SDIR), illustrated in Fig. 1(g), requires only one motor to drive within the pipe. This greatly simplifies the mechanical structure as well as the control system. However, just like creep-type robots, this type of robot also has the tendency of getting jammed inside the pipe. SDIRs are wall-pressed, which allows them to easily climb vertical pipes [16]. Kakogawa et al. presented a screw drive in-situ pipe robot that uses only two actuators to navigate through a bent pipe and T-branch between 109 and $129 \mathrm{~mm}$ of diameter. The robot has three different control modes. Screw-driving mode, for forward/backward movement; steering mode, to navigate through branch pipes or elbows, and roll mode, to change its navigation direction in pipes where it cannot steer [7].

A snake-type robot is shown in Fig. 1(h). It has multiple modules with terrain adaptability by means of wheel or foot locomotion. Thus, a snake-like robot has the greatest potential for application to industrial pipe inspection [17]. An SPC robot developed by Dai et al. is a spiral pipe-climbing robot that can expand or contract to vary its size as needed. It is able to adapt to pipes of different diameters. It is a wheeled multi-module structure, where each module can spin to better face other modules [5].

With the above features in mind, a combination of snakeand support-type robot is potentially the most suitable setup for industrial in-situ pipe inspection robots. This is due to the structure's flexibility and adaptability to move along a variety of pipe configurations: horizontal, vertical, reduction and bent pipes.

\section{PROPOSED DESIGN}

The robot architecture is based on the theoretical work of Douadi et al. [3], [4] and the preliminary design of Lamonde [18]. Their effort laid the foundation of the mechanical design and a computational framework of a 2-dimensional kinematics and dynamics of the robot. The concept consists of multiple (semi-)clone modules of identical dimensions. Each module is equipped with four independent arms with active 
shoulder joints. Each arm has an active wheel joint. The modules are connected through passive joints, as shown in Fig. 2.

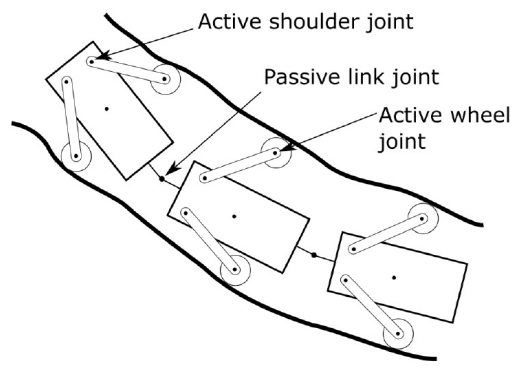

Fig. 2. General robot architecture

This multi-joint robot architecture has the advantage of offering enough degrees of freedom to escape possible singularities while the robot navigates along the pipe, especially around sharp turns [3], [4].

The robot concept shown in Fig. 2. does not take in consideration the physical components required to achieve the desired motion. A preliminary investigation by Lamonde [18] determined that a passive free-floating module would be needed to accommodate the power pack required for autonomous locomotion.

As a first prototype, a robot with three modules were built. The two modules at the front and back of the robot are driving modules, to help locomote the vehicle, whereas the middle module is the control module which carries the main electronics (Raspberry Pi, battery, etc.). Each driving module has four arms around the module that can be activated independently. A detailed breakdown of the module components is provided in Fig. 3 and Table I.

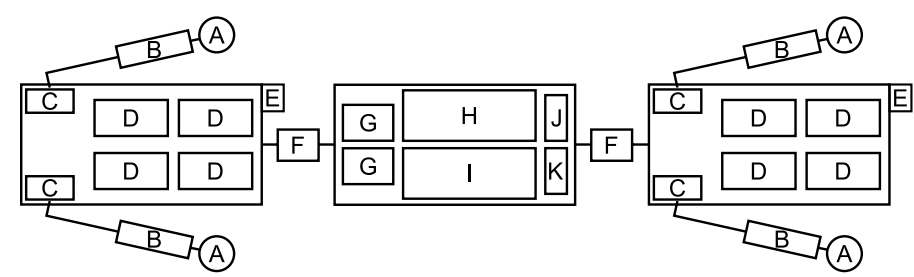

Fig. 3. Architecture of the prototype robot

The driving modules have two principal mechanisms: a worm drive at location $\mathrm{C}$ and a gear drive at location $\mathrm{B}$. The robot arms are driven by $180^{\circ}$ digital servomotors that move the worm screws which are meshed with the worm gears. Fig. 4 shows an exploded view of the propulsive module internal frame and the worm gear mechanism for a single arm. The propulsion is provided by a gearmotor coupled to a bevel gear. This right angle drive allows the gearmotors to be placed along the arm axis. The bevel gears and gearmotors have reduction ratios of $1: 2$ and 1000:1, respectively. The gear ratio was chosen to reduce the torque requirements while providing
TABLE I

COMPONENTS OF THE PROTOTYPE ROBOT

\begin{tabular}{l|l|l}
\hline Label & Part Name & Properities \\
\hline A & Wheel & Aluminum \\
B & Gearmotor & $1000: 1,32 \mathrm{rpm}, 0.8 \mathrm{Nm}$ \\
$\mathrm{C}$ & Worm gear mechanism & Alloy steel, ratio of $0.1: 1$ \\
$\mathrm{D}$ & Digital servomotor & $180^{\circ}, 1.3 \mathrm{Nm}$ \\
$\mathrm{E}$ & IMU & $6 \mathrm{DOF}$ \\
$\mathrm{F}$ & Universal joint & Max. operating angle: $45^{\circ}$ \\
$\mathrm{G}$ & Motor driver & $1.7 \mathrm{~A}$ output current \\
$\mathrm{H}$ & Raspberry Pi and servo HAT & Version 3B+ \\
$\mathrm{I}$ & Batteries & $7.4 \mathrm{~V}, 3 \mathrm{~A}$ \\
$\mathrm{~J}$ & Voltage converter for motors & $6 \mathrm{~V}, 15 \mathrm{~A}$ \\
$\mathrm{~K}$ & Voltage converter for Ras Pi & $5 \mathrm{~V}, 3 \mathrm{~A}$ \\
\hline
\end{tabular}

the required range of motion, and to fit inside the module body. The gearmotor ratio was selected with the peak torque required in the wheel shafts when there is a change in the diameter of the pipe. The peak torque is $0.43 \mathrm{~N} \cdot \mathrm{m}$ and it was obtained by simulation in [3]. Therefore, the minimal torque for the gearmotor is $0.22 \mathrm{~N} \cdot \mathrm{m}$ and the selected gearmotor has a peak torque of $0.8 \mathrm{~N} \cdot \mathrm{m}$. The arm component arrangement is shown in Fig. 5. At no-load, the drive produces a forward velocity of $2.76 \mathrm{~cm} / \mathrm{s}$.

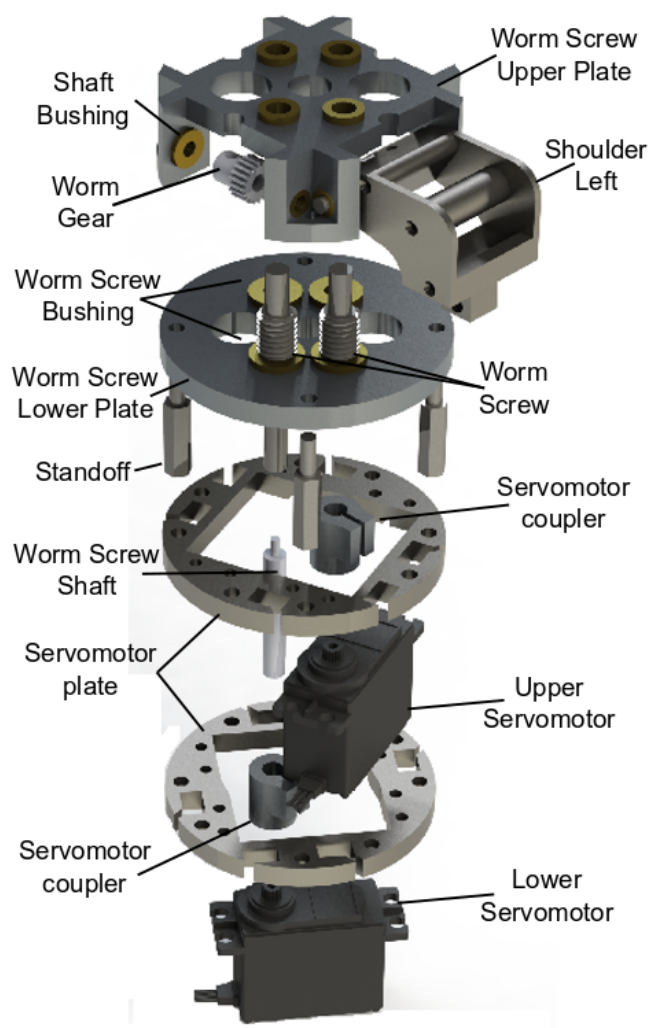

Fig. 4. Worm mechanism explosion

The arm shoulder holds the worm gear with a set screw. It can also hold a torsion spring to partially support the mass of the module. It is divided into two parts to facilitate the machining process while avoiding any deflection on the shoulder caused by the gear mechanism stress. 


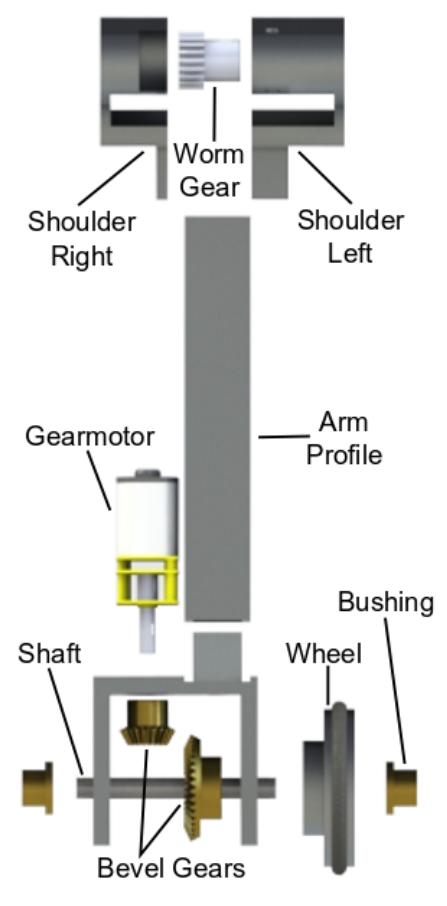

Fig. 5. Arm explosion

The driving module components are primarily machined from stock aluminum to avoid deflection in the structures due to the torque applied by the servomotor and the gearmotor. Non-load bearing components, such as the plate holding the servomotor and the complete control module, are made using a $3 \mathrm{D}$ printer to reduce the cost and weight in the fabrication process.

\section{Motion CONTROL}

The long-term goal of the project is to build an autonomous robot which can navigate, collect data, and generate reports about the anomalies along the pipe surface. To achieve full autonomy, a series of milestones need to be accomplished. As a first step, the motion control system is tested in open-loop mode. To that end, a bidirectional wireless communication link is established between the robot's main processing unit (Raspberry Pi) and a remote human-operated base computer. A graphical user interface (GUI) application is developed to run on the base computer to dispatch commands to the motors and to read and analyze data from the electronics aboard the robot. For example, signals from eight encoders connected to the propulsion gearmotors and two Inertial Measurement Unit (IMU) are transmitted regularly to the base computer. The power stage has eight motor drivers, one for each gearmotor, and one Servo HAT for the eight servomotors.

Fig. 6 depicts a block diagram of the electrical components aboard of the robot along with their interconnections. The control, analysis and communication protocols are written in Python. However, the data transfer is established via Secure
Shell (SSH). The GUI is displayed with Xming. The Servo HAT uses a PCA9685 integrated circuit which is an $\mathrm{I}^{2} \mathrm{C}$-bus with 16 channels. Each channel has its own 12-bit resolution (4096 steps) that operates in a programmable frequency of 24 to $1526 \mathrm{~Hz}$ with an adjustable duty cycle from 0 to $100 \%$. Thanks to the PWM adaptability in the output channels, half of the channels are programmed to control the servomotors within 0 to $180^{\circ}$. The other half is programmed to command PWM signals to the motor drivers. These are DRV8838 brushed motor drivers which enables the speed control of the motors with two inputs: Phase and Enable. The Phase pins comes from the Raspberry Pi to change the rotational direction of the motor depending of the received value. The Enable pins come from the Servo HAT. The received signal is a PWM pulse that varies the speed of the gearmotors from 0 to 32 RPM.

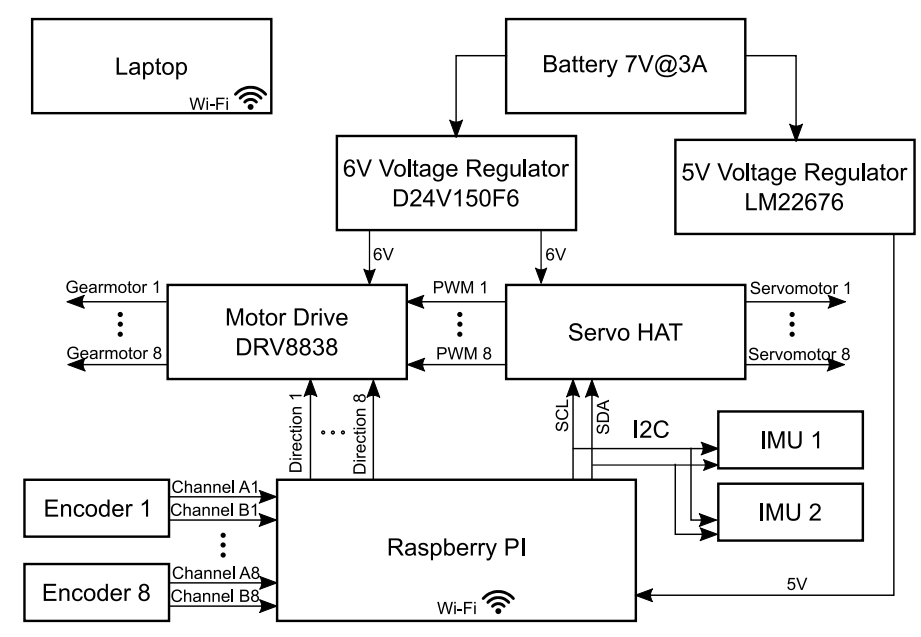

Fig. 6. Block diagram of the robot electronics

The IMU uses a MPU6050 chip with a dual 3-axis gyroscope and accelerometer. Since the robot naturally twists in the pipe the module's inclination must be determined to adjust its position inside the pipe. To do so, gravity measurements read by the accelerator are converted to inclination angles, as illustrated in Fig. 7. The figure shows the reference position and the rotation plane with the generated components and angles used to convert the gravity measurements into inclination angles [19]. The angles are calculated using:

$$
\begin{aligned}
& \theta=\operatorname{atan} 2\left(-A_{Y, \text { OUT }},-A_{Z, O U T}\right) \\
& \psi=\operatorname{atan} 2\left(-A_{X, O U T},-A_{Z, O U T}\right)
\end{aligned}
$$

where $A_{X, O U T}, A_{Y, O U T}$ and $A_{Z, O U T}$ are the IMU's angular accelerations about the $X, Y$, and $Z$ axes, respectively. The yield angles in (1) and (2) have an interval of $\left[-180^{\circ},+180^{\circ}\right]$, where $-180^{\circ}$ is the same as $+180^{\circ}$. This method assumes quasi-static or constant velocity and that the primary acceleration measurement is due to gravity. 


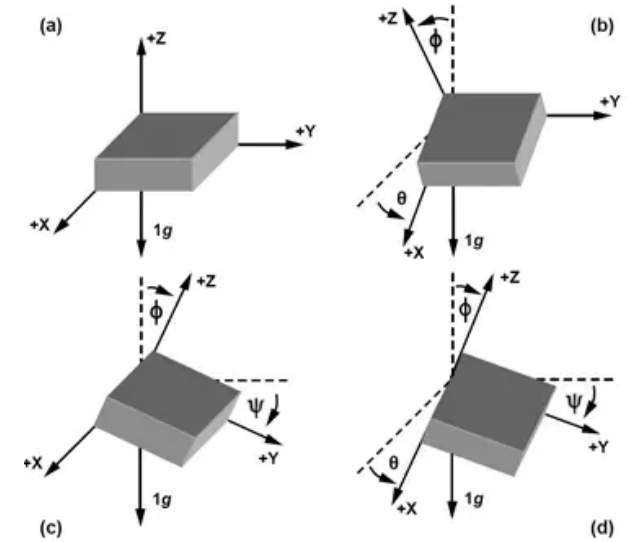

Fig. 7. Rotation planes with components and angles [19]

A flowchart of the communication protocol between the GUI and the robot is depicted in Fig. 8. This communication strategy is adopted to test individual sensors and actuators in open-loop control mode. It will be replaced at a later stage of the development by an autonomous feedback loop to track the robot's desired trajectory inside the pipe.

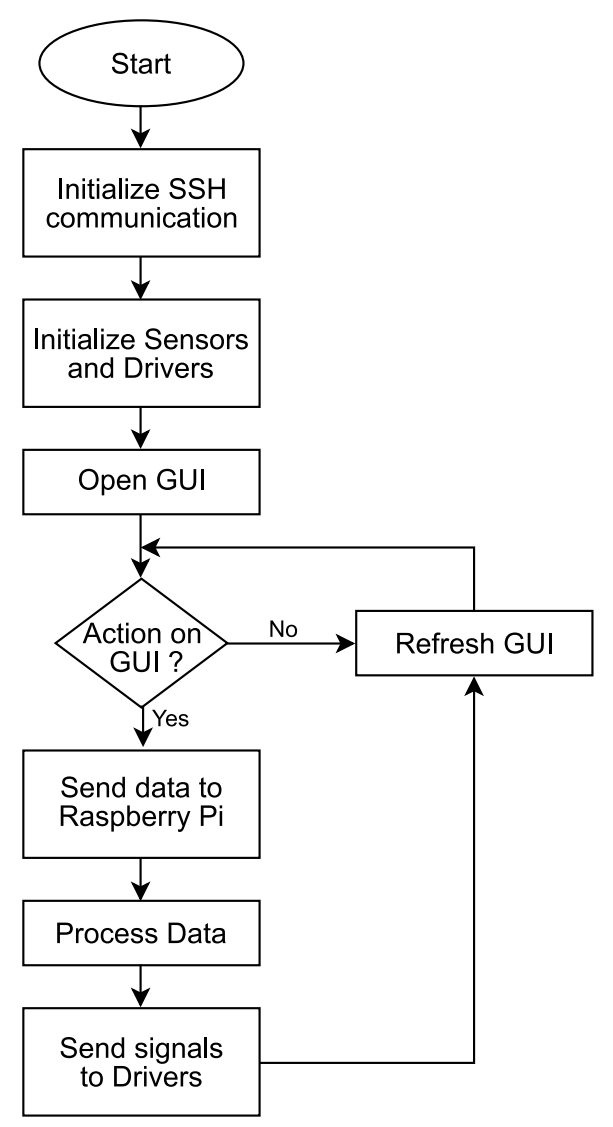

Fig. 8. Flowchart of the GUI's communication algorithm with the robot

\section{Assembly and Testing}

The prototype with two driving modules and one control module has a total length of $58 \mathrm{~cm}$ and a total mass of $2.75 \mathrm{~kg}$. The control module hosts two $1500 \mathrm{mAh} 2 \mathrm{~S} 25 \mathrm{C}$ lithium-polymer batteries, the on-board computer, the Servo HAT, voltage converters and the drivers for the gearmotors. It also carries two driving boards to hold the wires running through the module. Each of the two propulsive modules runs 13 cables distributed as follows: 2 for each gearmotor, 1 for each servomotor, and 1 for each IMU sensor.

Preliminary motion tests were conducted to run the robot back and forth along $84 \mathrm{~cm}$ inside a 6-inch clear pipe with an average speed of $2.47 \mathrm{~cm} / \mathrm{s}$. A photo shot of the setup is shown in Fig. 9.

A series of power consumption tests were performed on the prototype with and without load (the load is the weight of the robot). In the first test, all the electronics were turned on while the robot is at rest. In the second and the third test, the robot is set to, respectively, activate four or eight servomotors simultaneously. In the fourth and the fifth test, all eight gearmotors are set to operate at $50 \%$ and $100 \%$ of their full speeds, respectively. The last two tests were to activate all the actuators simultaneously at 50\% and $100 \%$ of the gearmotors full speed. The results of these tests are summarized in Table II.

The fourth and the fifth tests suggest that, when the gearmotors are being used at full speed the power consumption is less than at medium speed because at this rate the gearmotors demand less energy. The last two tests suggest that the robot must avoid the movement of the gearmotors at medium speed and the servomotors at the same time because this configuration consumes more energy than any other. However, this configuration could be performed during navigation through bent pipes or during the adaptation into a new pipe diameter which is less common in pipe inspections.

TABLE II

RESULTS OF THE POWER CONSUMPTION TESTS

\begin{tabular}{c|l|l|l}
\hline Test & Component & Current & Power \\
\hline 1 & On-board computer and drivers & $0.45 \mathrm{~A}$ & $3.37 \mathrm{~W}$ \\
2 & 4 Servomotors & $1.67 \mathrm{~A}$ & $12.52 \mathrm{~W}$ \\
3 & 8 Servomotors & $2.50 \mathrm{~A}$ & $18.75 \mathrm{~W}$ \\
4 & 8 Gearmotors at 50\% & $2.35 \mathrm{~A}$ & $17.62 \mathrm{~W}$ \\
5 & 8 Gearmotors at 100\% & $1.00 \mathrm{~A}$ & $7.50 \mathrm{~W}$ \\
6 & 8 Servomotors / 8 Gearmotor at 50\% & $2.60 \mathrm{~A}$ & $19.50 \mathrm{~W}$ \\
7 & 8 Servomotors / 8 Gearmotors at 100\% & $1.45 \mathrm{~A}$ & $10.87 \mathrm{~W}$ \\
\hline
\end{tabular}

To study the robot's endurance a last test was conducted. This test turned on all the electronics and actuators using a fully charged battery of $3000 \mathrm{mAh}$ at $7.4 \mathrm{~V}$. Then, the robot moved with full speed back and forth along the pipe. During this test the servomotors were driven from 0 to $180^{\circ}$ every 5 minutes. The robot was able to sustain this test for 54 consecutive minutes, which provides an idea of the autonomy time of the robot. 


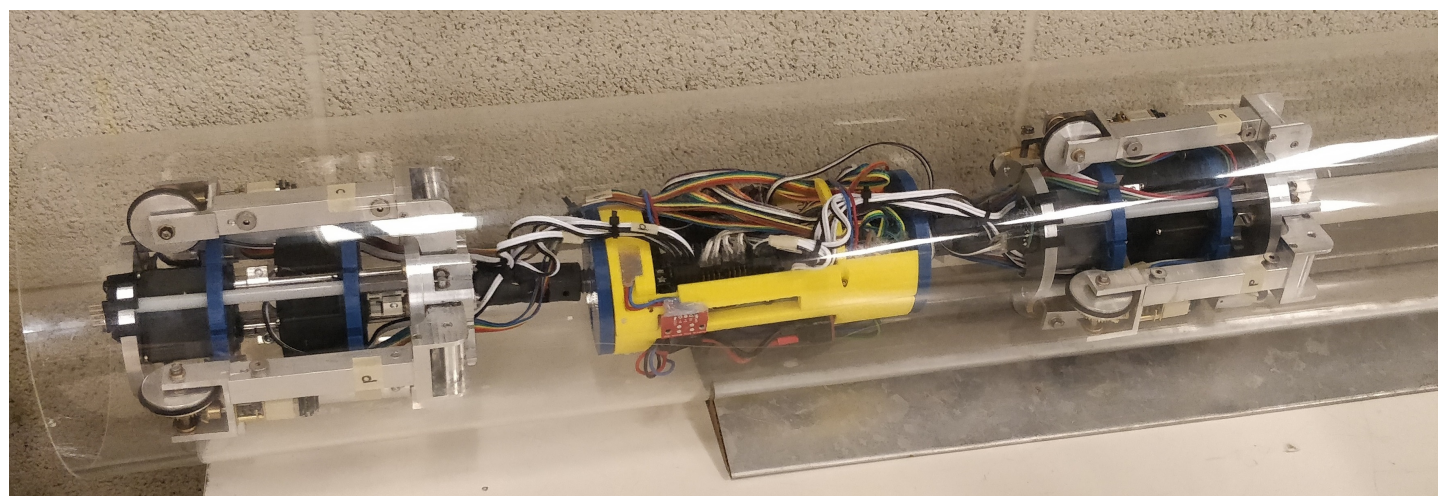

Fig. 9. Robot inside a 6-inch pipe

\section{CONClusion And Future WORK}

This work describes the first implementation and prototyping of a pipe inspection robot, as a part of a long-term project aiming at developing an autonomous robot capable of navigating and detecting faults inside gas pipelines with limited or no human intervention. The actual prototype is comprised of two traction and one control modules. The robot's locomotion was successfully tested in an open-loop motion control mode and tests also revealed that motion could be sustain for 54 minutes.

Planned future work includes developing a closed-loop trajectory tracking controller and integrating it with the robot's dynamic model. More effort is still needed to improve the robot's path planner to avoid singularities when negotiating sharp turns. More rigorous tests will also be conducted with different pipe diameters and configurations. Finally, because the controller and mechanisms allow the robot to have an odd number of modules. More research is needed to analyze the addition of modules to carry additional sensing instruments.

\section{REFERENCES}

[1] A. Alfano, "Extending the life of aging pipeline infrastructure," [Online]. Available: https://www.gptindustries.com/en/downloads/extending-lifeaging-pipeline-infrastructure. [Accessed: 11-Jan-2020].

[2] A. Reeves and J. Ryan, "Pipelines in canada," The Canadian Encyclopedia, June 2019.

[3] L. Douadi, D. Spinello, and W. Gueaieb, "Dynamics and control of a planar multibody mobile robot for confined environment inspection," Journal of Computational and Nonlinear Dynamics, vol. 10, September 2014, p. 011005.

[4] L. Douadi, D. Spinello, W. Gueaieb, and H. Sarfraz, "Planar kinematics analysis of a snake-like robot," Robotica, vol. 32, August 2013, pp. 659-675.

[5] J. Dai, Y. Xu, and W. Zhang, "Spc robot: A novel pipe-climbing robot with spiral extending of coupled differential," in 2017 IEEE International Conference on Robotics and Biomimetics (ROBIO), December 2017, pp. $1088-1093$.

[6] J. Okamoto, J. C. Adamowski, M. S. G. Tsuzuki, F. Buiochi, and C. S. Camerini, "Autonomous system for oil pipelines inspection," Mechatronics, vol. 9 (7), 1999, pp. $731-743$.

[7] A. Kakogawa, T. Nishimura, and S. Ma, "Development of a screw drive in-pipe robot for passing through bent and branch pipes," in International Symposium on Robotics, October 2013, pp. 1-6.
[8] Z. Song, H. Ren, J. Zhang, and S. S. Ge, "Kinematic analysis and motion control of wheeled mobile robots in cylindrical workspaces," IEEE Transactions on Automation Science and Engineering, vol. 13 (2), April 2016, pp. 1207-1214.

[9] M. A. A. Wahed and M. R. Arshad, "Wall-press type pipe inspection robot," in 2017 IEEE 2nd International Conference on Automatic Control and Intelligent Systems (I2CACIS), October 2017, pp. 185-190.

[10] I. N. Ismail, A. Anuar, K. S. Sahari, M. Z. Baharuddin, M. Fairuz, A. Jalal, and J. M. Saad, "Development of in-pipe inspection robot: A review," in 2012 IEEE Conference on Sustainable Utilization and Development in Engineering and Technology (STUDENT), October 2012, pp. 310-315.

[11] Y.-S. Kwon and B.-J. Yi, "Design and motion planning of a two-module collaborative indoor pipeline inspection robot," IEEE Transactions on Robotics - TRob, vol. 28, June 2012, pp. 681-696.

[12] Y.-G. Kim, D.-H. Shin, J.-I. Moon, and J. An, "Design and implementation of an optimal in-pipe navigation mechanism for a steel pipe cleaning robot," in 2011 8th International Conference on Ubiquitous Robots and Ambient Intelligence (URAI), November 2011, pp. 772-773.

[13] X. Yu, Y. Chen, M. Z. Q. Chen, and J. Lam, "Development of a novel in-pipe walking robot," in 2015 IEEE International Conference on Information and Automation, August 2015, pp. 364-368.

[14] Y. Zhang, M. Zhang, H. Sun, and Q. Jia, "Motion analysis of a flexible squirm pipe robot," in 2010 International Conference on Intelligent System Design and Engineering Application, October 2010, vol. 1, pp. 959-963.

[15] K. Xu, H. Xu, Q. Yu, Z. Wang, and W. Xu, "A novel crawling in-pipe robot design," MATEC Web of Conferences, vol. 61, January 2016, p. 04017.

[16] R. Tao, L. Qingyou, L. Yujia, and C. Yonghua, "Basic characteristics of a novel in-pipe helical drive robot," Int. J. of Mechatronics and Automation, vol. 4, January 2014, pp. 127 - 136.

[17] K. Wang, W. Gao, and S. Ma, "Snake-like robot with fusion gait for high environmental adaptability: Design, modeling, and experiment," Applied Sciences, vol. 7, November 2017, p. 1133.

[18] J. Lamonde, Development of a Multi-Body Autonomous Inspection Robot for Small Diameter Pipes, Master's thesis, University of Ottawa, 2017.

[19] C. J. Fisher, "Using an accelerometer fon inclination sensing," Convergence Promotions, May 2011, [Online]. Available: https://www.digikey.com/en/articles/techzone/2011/may/using-anaccelerometer-for-inclination-sensing. [Accessed: 28-Dec-2019]. 